\title{
AFTERIMAGE MOVEMENT DURING SACCADES IN THE DARK
}

\author{
O.-J. Grüsser, A. KrizIĆ and L.-R. Weiss \\ Department of Physiology, Freie Universität Berlin, Arnimallee 22, D-1000 Berlin 33. F.R.G.
}

(Received 7 October 1985; in revised form $10 \mathrm{July} \mathrm{1986)}$

\begin{abstract}
The spatial values of retinal coordinates are "recalibrated" to the "egocentric" coordinates during and after a saccade within a fraction of a second. We measured the time constant of this retinal coordinate transformation by means of an afterimage technique: our ten subjects performed "auditory" horizontal saccades in total darkness $(0.2-4.5$ saccades $/ \mathrm{sec})$. At a saccade frequency below $1 \mathrm{saccade} / \mathrm{sec}$, the subjects observed saccadic displacement of the foveal afterimage, but the afterimage seemed to arrive at its final position more slowly than the center of gaze (state 1). At saccade frequencies above 1.5 saccades/sec, the perceived amplitude of afterimage displacement decreased with increased saccade frequency (state 2). Above 2 saccades/sec all subjects perceived $t$ wo stationary afterimages simultaneously at the saccadic end-position (state 3). A further increases in saccade frequency reduced the distance between the two afterimages till only one stationary afterimage was seen in a mid-position between the two auditory targets at a saccade frequency above $3.2-3.5$ saccades/sec (state 4). Saccade amplitude remained constant within the frequency range between 0.2 and 4.5 saccades/sec. A one-step or two-step linear model was applied to simulate the experimental data, indicating that the spatial coordinates shift more slowly than the saccadic eye movements.
\end{abstract}

Spatial values of retinal coordinates Saccades Efference copy Afterimage Migrainephosphenes

\section{INTRODUCTION}

When one observes a lasting foveal afterimage, i.e. an image perfectly stabilized on the retina, in the dark or against a homogeneous background, it is seen moving in the extrapersonal space whenever the position of gaze is changed by voluntary saccades or eye pursuit movements. This observation was first reported by Aristotle who wrote in his essay, "On dreams": "And if we look at the sun or any other bright object and close thereafter our eyes, then when our line of sight is directed straight ahead, we see first an image of the same colour, whereever the center of gaze moves...".

Evidently the apparent motion of the afterimage is correlated to the change in spatial values of the retinal coordinates during voluntary eye or gaze movements. This "recalibration" or coordinate transformation process (Bell, 1823; Purkinje, 1825a, b; Hering, 1861, 1879; Mach, 1886) is not necessarily linearly correlated with the actual gaze position. When paying attention to the afterimage movement during large voluntary saccades in the dark, one gains the impression that the afterimage changes its position much more slowly than the center of gaze (Purkinje, 1825a, b).
Thus the adjustment in the spatial values of the retinal coordinates during and after saccadic eye movements seem to be delayed with respect to the change in the actual center of gaze position. Purkinje also observed that very fast horizontal back and forth saccades lead to a corresponding apparent movement of the stationary objects (oscillopsia).

Several components might contribute to the recalibration of retinal coordinates during and after saccades:

(a) Efference copy signals representing the motor commands of change in gaze position.

(b) Afferent mechanoreceptor signals originating in the eye muscles, tendons or other structures of the orbita.

(c) Retinal movement or displacement signals.

In the following we applied a method by which all retinal displacement signals were absent, since an absolutely stabilized retinal stimulus was applied. Voluntary horizontal "auditory"saccades were produced in total darkness towards acoustic targets, and the frequency of the subject's horizontal back and forth saccades was increased in steps by increasing the alter- 

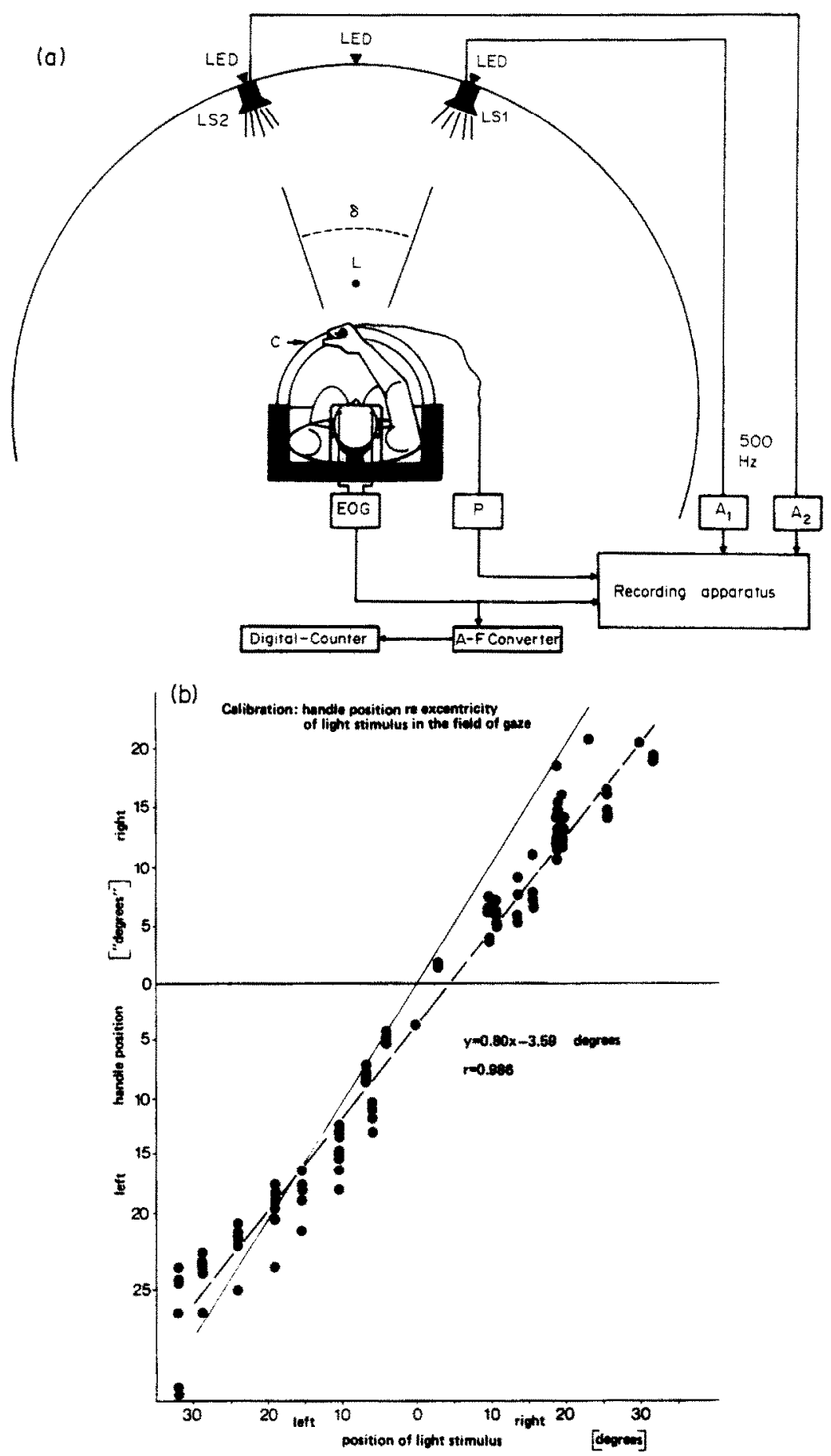

Fig. 1. (a) Scheme of the experimental set-up. The subject sat in the center of a vertical cylinder, $280 \mathrm{~cm}$ in diameter, with his head tightly fixed. Two loudspeakers (LS1, LS2) were placed on the wall of the cylinder at eye level and an angular distance $A$. Three light-emitting diodes (LED) served for calibration of the DC-electrooculogram. Before the measurements the subjects had to fixate a small strong light source or a photoflash $L$ monocularly to imprint a long foveal afterimage. When looking at the sounding loudspeakers in total darkness, the subject perceived a shift $\mathrm{d}$ in the afterimage position and "pointed" with the movable handle on a rail $(C)$ towards the respective end-position of the afterimage. This position signal $P$ was recorded simultaneously with the $D C$-electrooculogram and the loudspeaker signals $\left(A_{1}, A_{2}\right)$. (b) Calibration of the pointing device: in otherwise total darkness a small light spot of 0.1 deg diameter was projected at eye level onto the cylinder wall at different horizontal eccentricities (abscissa). The subject had to fixate the target and point to the spot of light with the handle using his right hand. Note the slight left/right off-set; the average slope was smaller than 1.0. The dashed line gives the regression line of the data; the continuous line represents the linear relation between light position and handle position at gain I without left/right off-set. 
nation rate of the acoustic stimuli. The subjects reported on movement and displacement amplitude of a small foveal afterimage. From our data the recalibration time course of spatial retinal coordinate values can be estimated. A short report has already been presented at an earlier meeting (Grüsser and Krizič, 1984).

\section{METHODS}

The experiments were performed in 10 adult subjects ( 4 female, 6 male) between 26 and 52 years of age with normal vision and no overt oculomotor or vestibular impairment. Myopia was compensated by eye glasses for the imprinting of the afterimage. All subjects were unpaid volunteers. Seven of them were uninitiated and had never participated in oculomotor or visual psychophysics experiments; three were experienced observers. Except for the somewhat higher frequency limit of horizontal saccades in the experienced observers, no essential differences were found in the experimental data. The naive subjects were not acquainted with the purpose or theory of the experiments, while the experienced observers were.

\section{Eye movement recordings}

The subjects sat in a comfortable chair with a chin rest and a headholder placed tightly against the forehead and occiput [Fig. 1(a)]. Possible head movements (rotation) were thus reduced to a minimum ( $<1 \mathrm{deg})$. The subjects were also instructed not to move the head voluntarily. In the performance of back and forth saccades at the upper frequency limit between 3.0 and $4.5 \mathrm{saccades} / \mathrm{sec}$, the gaze movement commands led involuntarily to head movements, however actual movements were mechanically restricted by the headholder.
Horizontal eye movements were recorded by means of DC-electrooculography (EOG) with conventional amplifiers (frequency range 0-100 or $0-30 \mathrm{~Hz}$, gain $300-1000$, precision of amplitude measurements about $0.5 \mathrm{deg}$ ). Optoelectronic coupling separated subject and recording device.

\section{Afterimage and auditory saccades}

In earlier experiments a monocular foveal afterimage was produced by fixation of a small $150 \mathrm{~W}$ DC-bulb placed about $100 \mathrm{~cm}$ from the eye. The diameter of the afterimage produced by this device was about $0.1-0.2 \mathrm{deg}$, depending on the precision of fixation during the $5-8 \mathrm{sec}$ imprinting period. In more recent experiments the afterimage was produced by a short $(<0.1 \mathrm{msec})$, high-intensity photoflash $(0.05 \times$ $0.3 \mathrm{deg}$ vertical light slit). The afterimage was seen in darkness for about 2-5 min. After it had disappeared, a second afterimage was imprinted using the other eye. The subject sat in the center of a vertical cylinder $280 \mathrm{~cm}$ in diameter and $200 \mathrm{~cm}$ high. On the wall of the cylinder two loudspeakers were fixed at the subject's eye level [Fig. 1(a)]. The horizontal distance between the loudspeakers could be varied. This distance is given in degrees of visual angle with respect to the subject's "cyclopean eye". Three small lightemitting diodes (diameter $0.08 \mathrm{deg}$ ) were placed horizontally $19 \mathrm{deg}$ apart in the wall of the cylinder at the subject's eye level for the purpose of EOG calibration, performed every $30-80 \mathrm{sec}$.

Alternating $500 \mathrm{~Hz}$ square wave signals of equal duration and an intensity of about $50 \mathrm{~dB}$ SPL were produced by the two loudspeakers $(6 \mathrm{~cm}$ dia, Fig. 2). Due to a slight difference in signal transfer characteristics the subjects could detect from the sound location as well as from the sound quality which loudspeaker was turned

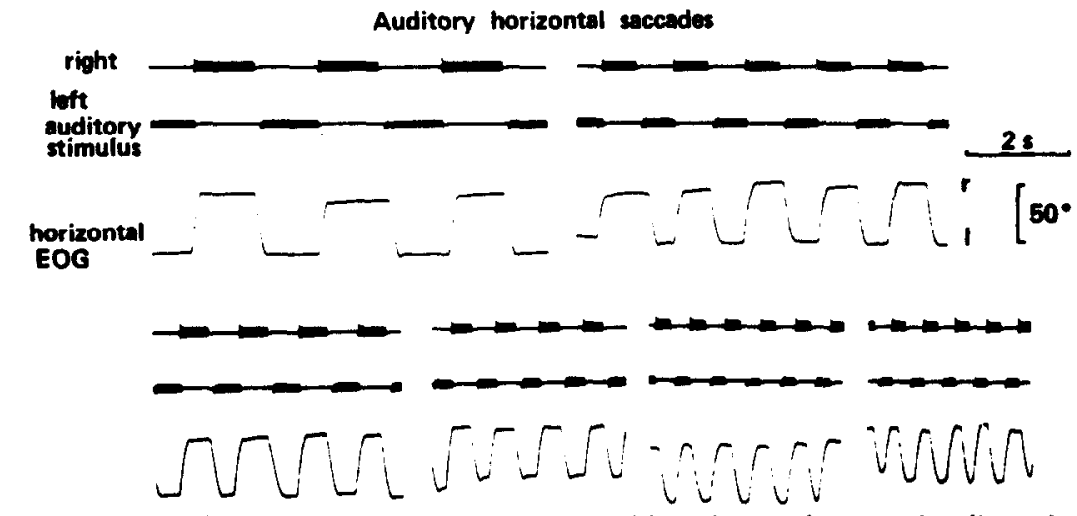

Fig. 2. EOG-recording example of horizontal eye position changes by saccades directed towards the sounding auditory targets (Fig. 1). Six different alternation frequencies $(0.45-3.6$ saccades/sec). 
on. The subjects were asked to look at the sounding loudspeaker as quickly as possible. Since the alternation sequence between the loudspeakers was periodic and therefore predictable, at low alternation frequencies the subjects performed "anticipatory" saccades, i.e. the latency of the saccades as compared to the loudspeaker signals was found to be near zero. In the present study, however, we were only interested in obtaining a regular voluntary horizontal saccade sequence, so we did not concern ourselves further with this matter. The alternation frequency of the saccades and saccade amplitude were measured from the EOGrecordings.

Subject instruction and the measurement of perceived afterimage position

At a low saccade frequency $\quad(\leqslant 0.3$ saccades $/ \mathrm{sec}$ ) all subjects could easily see the movement or change in position of the foveal afterimage. The task was explained at such low stimulus alternation frequencies: the subjects were asked to move a handle along a plastic semicircular sector in front of them and to "point" with the handle in the direction of the perceived end-position of the afterimage on the right side and, after a further 5-10 saccades, on the left side [Fig. 1(a)]. The handle was moved along a cogwheel band and rail [C in Fig. 1(a)] fixed to the semicircular sector. Thus the handle position could be recorded directly using a simple potentiometer circuit. A small notch placed in front of the subject indicated the mid-position of the handle. When the subjects were asked to point to a projected small spot of light ( $0.08 \mathrm{deg}$ diameter), a linear relationship between horizontal eccentricity of the light spot in the field of gaze and the deviation of the handle from the mid-position was found. The gain of this relationship amounted to $0.8-0.95$ [Fig. 1(b), cf. Bock and Eckmiller, 1983]. Thus pointing with the handle measured in degrees of visual angle can be taken as a relative and linear measurement of eccentricity perceived in the field of gaze. It should be noted, however, that although the slope between angle of handle pointing and horizontal eccentricity of gaze was between 0.8 and 0.95 with real light stimuli, it was less in most subjects in the afterimage experiments. This finding will be discussed in a later report (Beyer and Grüsser 1986). Nevertheless, pointing eccentricity was also linearly correlated in the afterimage experiments to ec- centricity of the center of gaze from the midline (head fixed).

The horizontal DC-EOG, auditory signals and handle position signals were recorded on a paper oscillograph (modified Siemens Cardirex).

In the main series of experiments the horizontal separation of the two loudspeakers was fixed at $39 \mathrm{deg}( \pm 19.5 \mathrm{deg}$ eccentricity). Data were obtained in 10 subjects with this paradigm. In two subjects the symmetrical loudspeaker separation was varied systematically and in one subject the loudspeakers were asymmetrically placed. The measurements lasted about 40-60 min for one set of data with a fixed loudspeaker distance. Longer experimental sessions were avoided due to subject fatigue. Thus. in the experimental series in which the distance of the loudspeakers and the alternation frequency of the horizonal saccades were varied systematically, 4 (subject J. S.) and 9 (subject O.-J.G.) sessions of about $1 \mathrm{hr}$ duration were necessary to collect the data.

\section{Data analysis and model computation}

Within the frequency range measured, the average alternation frequency of horizontal saccades corresponded in an attentive subject to the alternation frequency of the two loudspeakers. Once this state was achieved the amplitude for 10-20 successive horizontal saccades was measured for each alternation frequency. The algebraic mean of these measurements was used in further computation. The apparent shift in the horizontal afterimage to the left and to the right was measured once or twice for each saccade frequency. For computing averages the first measurement was used. As a rule, the second measurement deviated less than $10 \%$ from the first. For the computation of the mathematical models (pp. 222, 223) a digital computer (HP $1000 \mathrm{MX}$ ) was applied. Model computations were performed on individual as well as average data of 10 subjects.

\section{RESULTS}

The perceived movement amplitude of the afterimage depends on saccade frequency

Figure 2. exhibits recording examples of horizontal saccades elicited by the auditory stimuli placed $39 \mathrm{deg}$ apart horizontally. Accuracy of saccades can be seen from the standard error values displayed in Fig 4(a). Six different stimulus alternation frequencies and the correspond- 
ing saccades are displayed. In all subjects the angular size of the auditory saccades was larger than the angular distance of the auditory targets [cf. also Fig. 5(a,b)]. We were familiar with this observation from earlier experiments in which saccades evoked by auditory stimuli in the dark were measured (Gottschalk, Grüsser and Lindau, 1978; Grüsser, 1983). Only for very large horizontal or vertical saccades $(>80 \mathrm{deg}$ ) did the auditory saccades approximate the distance of the auditory targets (in degrees of visual angle). Pointing towards the auditory target with the hand and index finger resulted in

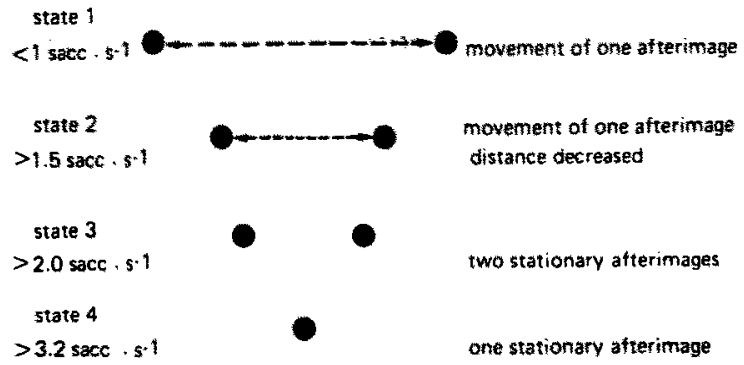

Fig. 3. Illustration of the four states of afterimage perception during horizontal auditory saccades performed at different rates as indicated. For further explanation, see text.
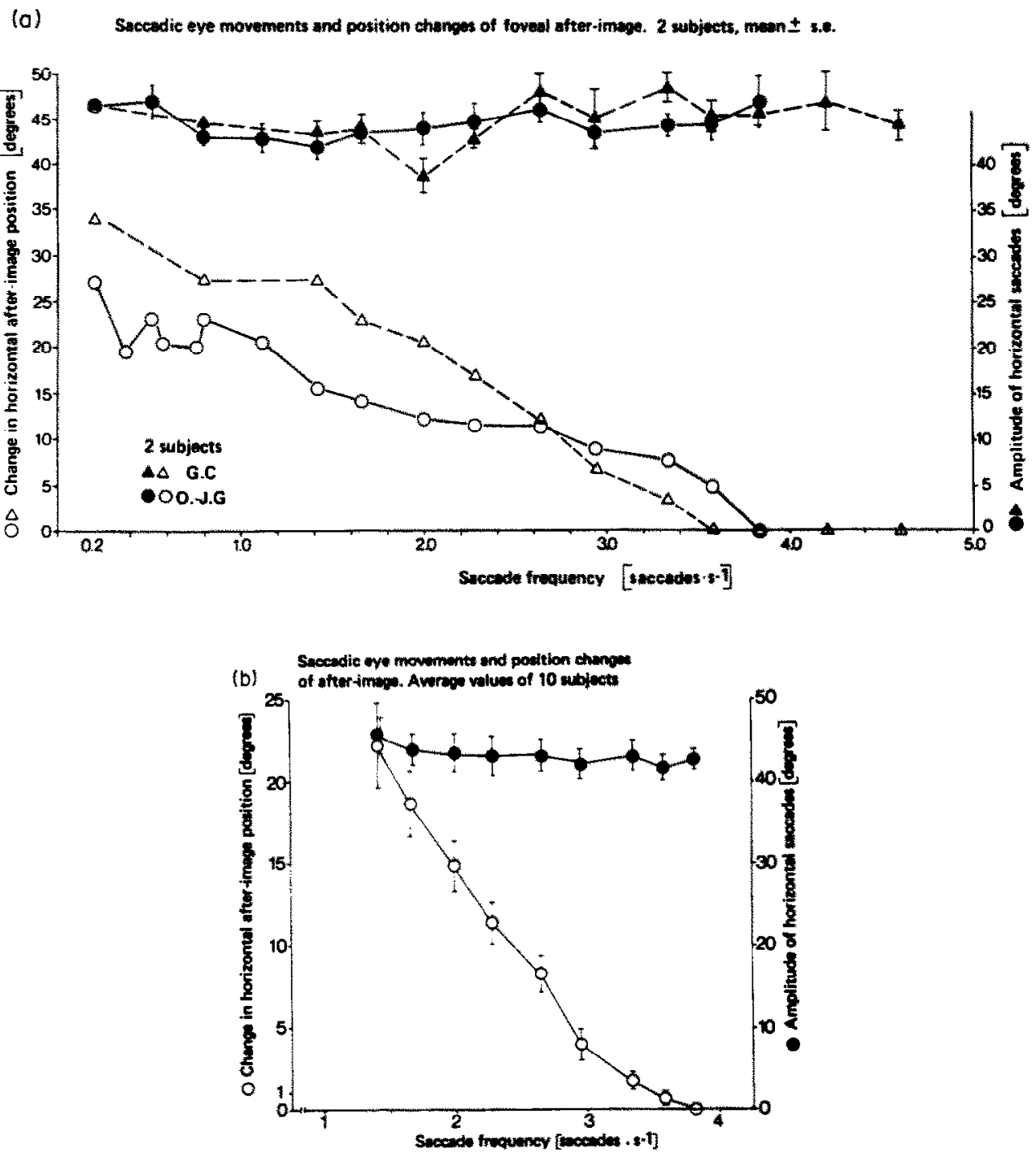

Fig. 4. (a) Relationship between saccade frequency (abscissa), amplitude of horizontal saccades (ordinate) and perceived change in horizonal position of a small foveal afterimage (ordinate, left side, open symbols). Data from two subjects. Note that amplitude of horizontal saccades barely changes with saccade frequency. (b) Same relationship as in (a), but algebraic mean and error of the mean obtained in 10 subjects. Note different scaling of the ordinate for saccade amplitude and afterimage displacement. 

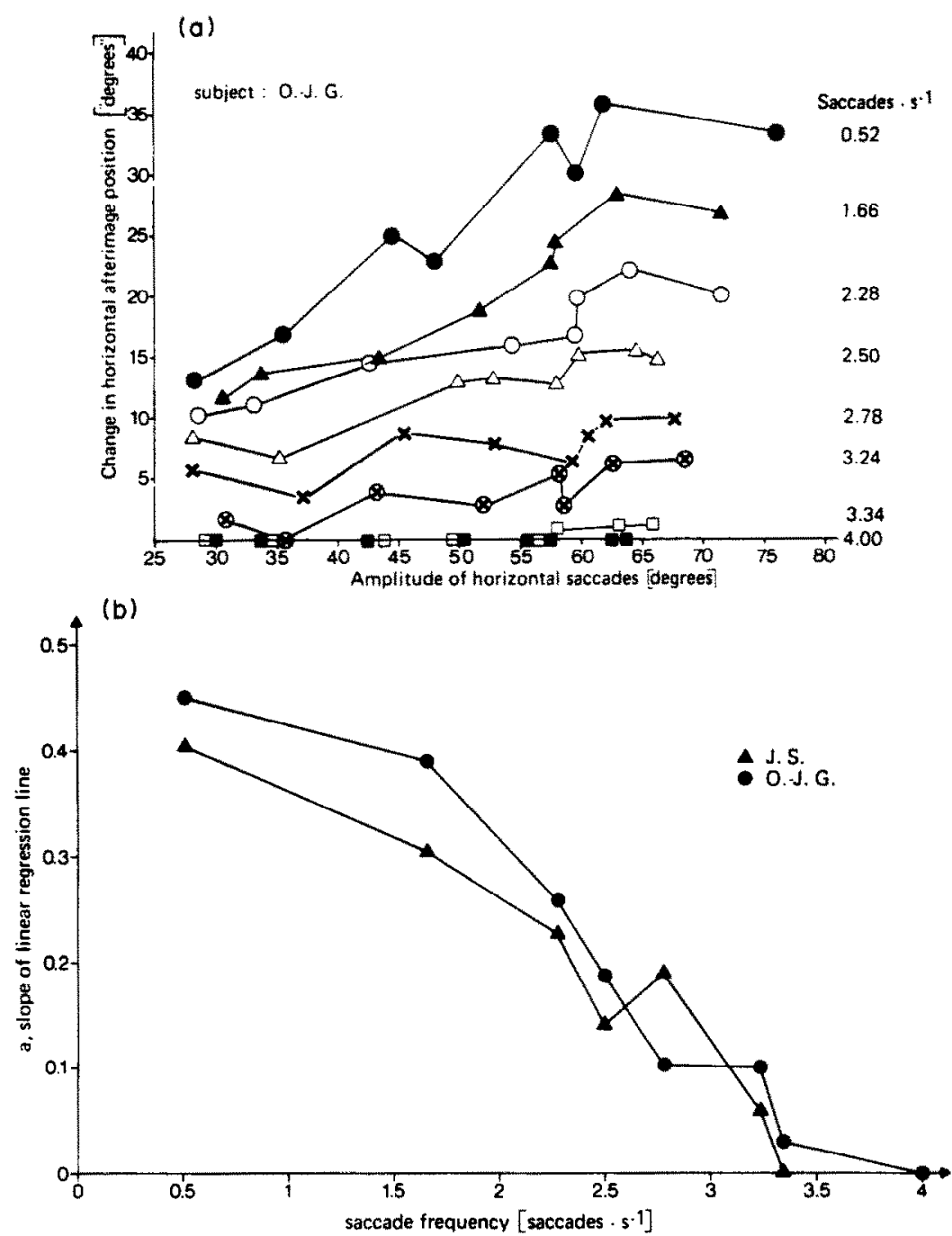

Fig. 5. (a) Relationship between change in afterimage position (ordinate) and amplitude of horizontal "auditory" saccades performed at different saccade frequencies. Data obtained in different sessions with one subject. (b) The slope $a$ of the linear regression line between change in horizontal afterimage position $d$ and amplitude $A$ of horizontal saccades was computed for different saccade frequencies [equation (1)] and is plotted as a function of saccade frequency (abscissa). Data obtained in two different subjects at 8 different saccade frequencies.

considerably less error. Either pointing was correct or overestimation of auditory target distance was less than $10 \%$.

With saccade alternation frequencies smaller

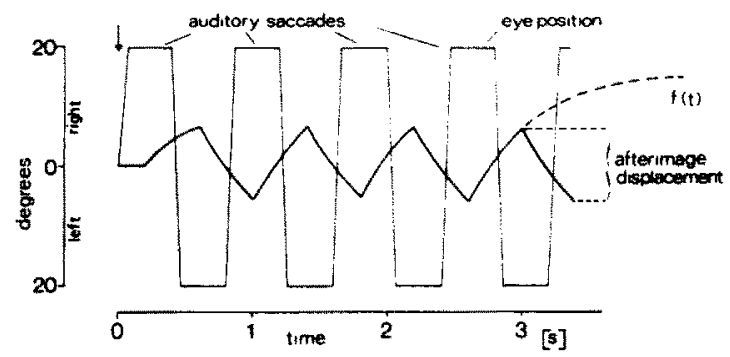

Fig. 6. Scheme to illustrate how recalibration of retinal coordinate spatial values depends on frequency of horizontal alternating saccades. than 1.2 saccades $/ \mathrm{sec}$ the subjects were always aware that they performed "good" saccades corresponding to the rhythm and amplitude of the auditory signals, whereas at a saccade frequency above 2 saccades/sec none of the subjects was sure about his performance. Even the more experienced had the impression that in a frequency range above 2.5 saccades/sec their saccades were not precise and probably much smaller than the acoustic target distance. Thus they were surprised to see from their EOG recordings that up to a frequency range between 3.8 and 4.5 saccades/sec the auditory saccades changed very little in their amplitude, if at all, as compared to the low frequency range [Figs 2 and $4(a, b)]$. Above a saccade frequency of 
2.5 saccades/sec the subjects reported that a fair amount of effort was required to fulfill the task.

The change in afterimage position at a saccade frequency of 1.5 saccades/sec was easily observed by all subjects. Most of them reported having the impression that the center of gaze was on target earlier than the afterimage, i.e. it seemed that their eyes moved faster than the afterimage (state 1, Fig. 3). When pointing in the direction of the perceived end-positions of the afterimage, all subjects moved the handle to considerably lower eccentricities $(50-85 \%)$ than those of the eye at the respective end-positions. When the alternation frequency of the auditory stimuli was increased, leading to a corresponding increase in the saccade frequency, the amplitude of the apparent horizontal shift in the afterimage diminished [state 2, Figs 3, 4 (a,b)]. Moreover, at a saccade frequency between 1.8 and 2.4 saccades/sec all subjects suddenly perceived two afterimages simultaneously, one on the left and one on the right side of the field of gaze (state 3 in Fig. 3). Either these two afterimages did not seem to move at all during the saccades or the subjects were undecided. With a further increase in saccade frequency the distance between the two afterimages decreased and at a saccade frequency above 3.5 saccades/ sec all subjects saw only one stationary afterimage in a position corresponding approximately to the midline in front of them (state 4). The reports of all subjects were very uniform and the data from experienced as compared to inexperienced subjects exhibited only minor differences [Fig. 4(a,b)].

\section{The effect of varying the horizontal separation of the auditory targets}

In two of the 10 subjects additional measurements were performed to find out whether the time course of recalibration of retinal spatial values depended on saccadic amplitude. The paradigm described in the preceding section was again applied, but the horizontal separation between the loudspeakers varied. In both subjects essentially the same results were obtained: at low alternation frequencies the saccade amplitude increased approximately linearly with the separation of the auditory targets, as did the apparent shift in afterimage position. Thus a linear relationship between saccade amplitude $A$ and amplitude $d$ of the shift in afterimage position was found [equation (1), Fig. 5(a)]. When the saccade frequency was increased above about 2 saccades/sec, a paradoxical effect was reported: the subjects experienced greater difficulty in performing fast back and forth horizontal saccades with small amplitudes than with larger ones. The frequency at which the afterimage doubled and showed reduced position change depended very little on the saccade amplitude or the horizontal distance of the auditory targets. The same was true for the saccade frequency at which the afterimage did not seem to move.

Due to the diminished apparent afterimage movement at saccade frequencies above $1.4 \mathrm{saccades} / \mathrm{sec}$, the relationship between amplitude of the horizontal auditory saccades $A$ and the change in horizonal afterimage position $d$ was greatly influenced by the saccade frequency [Fig. 5(a)]. From the relationship between $A$ and $d$ we computed the respective linear regression lines obtained at different saccade frequencies

$$
d=\underline{a} A+b \text { [degrees] }
$$

and plotted the slope $a$ as a function of saccade frequency [Fig. 5(b)]. This relationship characterizes the decrease in horizontal afterimage position change with increasing saccade frequency, independent of the respective saccade amplitude. It can be taken as another measure of the average time-course of retinal spatial coordinate recalibration generalized for saccade amplitudes between 25 and $70 \mathrm{deg}$.

\section{Lateral displacement of the auditory targets}

Next we asked whether the time-course of recalibration of retinal spatial coordinates depended on the field of gaze across which the saccades were performed. To answer this question we used one experienced subject and displaced the loudspeakers from a symmetrical position around the center of the field of gaze to a more asymmetrical position. This horizontal shift in loudspeaker position did not have any essential effect, however, on the outcome of the experiment. The same phenomena as described above were found: doubling of the afterimage with increasing saccade frequency above 2 saccades/sec, reduction in the distance of the two afterimages with further increase in saccade frequency and finally "stabilization" of one afterimage seen at a position in the extrapersonal space which corresponded to the midposition between the two auditory targets (saccade frequency $\geqslant 3.2 \mathrm{saccades} / \mathrm{sec}$ ). This rule was valid, irrespective of the direction and amount of horizontal displacement of the mid- 
line between the two loudspeakers up to $25 \mathrm{deg}$ from the center of the field of gaze.

\section{Extrafoveal afterimages}

When one gazes rapidly back and forth by means of large saccades across a structured visual surround ("voluntary nystagmus"), oscillopsia appears to be strong around the center of the field of gaze, while the apparent movement of the objects is less prominent in the visual field periphery. This phenomenon could be caused, of course, by retinal or central visual mechanisms correlated to the increase in receptive field diameter with retinal eccentricity. It could, however, also be caused by a different timecourse of recalibration of spatial retinal values. Based on this observation we performed in one experienced subject an experiment in which the afterimage was imprinted at a position about $5 \mathrm{deg}$ lateral to the left or right of the fovea and the same procedure was followed as mentioned above. Essentially the same observations were made as with foveal afterimages, except that at a saccade frequency above 3.4 saccades/sec the afterimage apparently remained stationary in a position deviating from the midline. The lateral displacement corresponded approximately to the displacement of the afterimage from the fovea.

Position changes of a migraine phosphene during auditory saccades; three chance observations

One of the authors was twice able to perform an experiment with a migraine phosphene. It was a typical "fortification" phosphene (Jolly, 1902; Lashley, 1941; Richards, 1971; Bücking and Baumgartner, 1974; Baumgartner, 1977; Jung, 1979) exhibiting a flickering rate of about $10 \mathrm{~Hz}$. The observation with one of the phosphenes shall be described in the following:

This flickering migraine phosphene began in the upper left quadrant of the visual field at about $50 \mathrm{deg}$ eccentricity and moved slowly towards the foveal region. This type of migraine phosphene was exceptional; the other $7 \mathrm{mi}$ graine phosphenes experienced by the subject over the last 8 years had all begun in the foveal region and moved outwards in the visual field. The experiments could be prepared before the migraine phosphene had reached the left half of the fovea, but no EOG recordings were possible since the observation was not made in the laboratory. Auditory stimuli at eye level and about 60 degrees horizontal distance were applied in the dark, and all phenomena of states
1 to 4 described for a retinal afterimage could be observed in this migraine phosphene. In a second "run" the fast horizontal saccades were repeated at different alternation frequencies (maximum about 4 saccades $/ \mathrm{sec}$ ) but with an additional afterimage about 0.2 deg in diameter placed into the fovea center and located about $2 \mathrm{deg}$ from the migraine phosphene. Under these conditions migraine phosphene and afterimage moved together, exhibited states 1 to 4 as described above and showed no movement relative to each other, i.e. they always seemed to move parallel to each other, regardless of the alternation frequency in state 1 or 2, while in state 3 two afterimages and two migraine phosphenes were observed.

These observations could be confirmed by one of our co-workers who suddenly observed a parafoveal (about 2 deg eccentricity) migraine phosphene after working the entire night in the laboratory. One of the authors immediately performed the same experiment with the subject as described above but used alternating finger snapping as auditory stimuli in the totally dark room (horizontal distance of the auditory targets about $60 \mathrm{deg}$ ). The subject reported a significant decrease in phosphene distance with increasing snapping frequency, at about 2 saccades/sec doubling of the phosphene and finally stillstand of the nearly foveal migraine phosphene at a saccade alternation frequency of about 3 saccades $/$ sec.

\section{MODEL COMPUTATIONS}

Let us assume that the amplitude of the horizontal back and forth saccades is $A$ degrees and the center of gaze position "straight ahead" (i.e. at the center of the field of gaze) is defined by the coordinates $0 ; 0 \mathrm{deg}$. Let us further assume that the spatial values of retinal coordinates change with the beginning of the neuronal command leading to a goal-directed voluntary saccade according to an arbitary time function $f(t)$. One can then easily deduce how the perceived shift in afterimage position $d$ depends on saccade frequency. In the diagram of Fig. 6 it is assumed that-as in our experiments-the center of gaze starts from the midline position, the first saccade points towards the right loudspeaker and the following saccades shift goaldirected horizontally between the right and the left loudspeaker according to the auditory signals. The value which the retinal spatial coordinates have reached by the time the command for 
the next backwards saccade interrupts the transformation process depends on $f(t)$ and on $t$. With a bit of algebra one can easily find $d$

$$
d=f(t) /[2-f(t)] \text { [degrees]. }
$$

Equation (2) is a general solution valid for an arbitrary set of monotonic functions, leaving the function $f(t)$ open for further explorations. Applying a digital computer program we substituted two simple exponential functions for $f(t)$, one describing a one-stage, the other a twostage linear filter

$$
\begin{aligned}
& f(t)=1-\exp \left[\left(-t+T_{1}\right) / \tau_{1}\right] \\
& f(t)=\left\{1-\exp \left[\left(-t+T_{2}\right) / \tau_{2}\right]\right\}^{2}
\end{aligned}
$$

$f_{1}(t)$ or $f_{2}(t)$ were used to find the time constants $\tau_{1}$ or $\tau_{2}$ and the dead time $T_{1}$ or $T_{2}$ of equation (2) leading to an optimum fit between experimental data and mathematic model. Examples for one set of data are show in Fig. 7(a,b). It should be pointed out that equations (3) or (4) both fitted the experimental data reasonably well but similarly a hyperbolic function or any other polynom could also be substituted. Exponential functions of time, however, have the advantage of being not only simple but also attributable to functions of neuronal networks operating according to the principle of "leaky integrators".

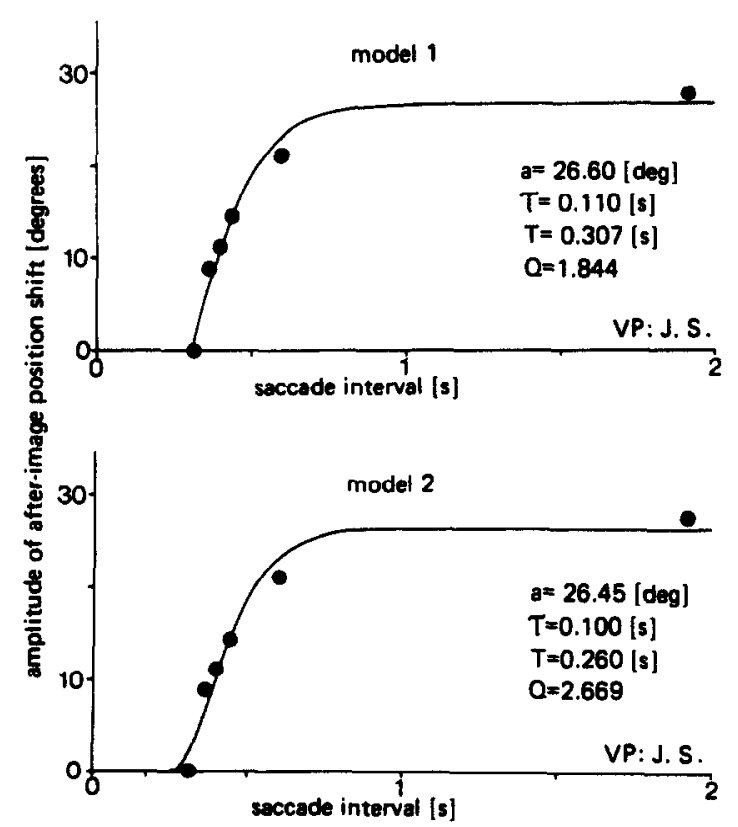

Fig. 7. (a) Change in afterimage position (ordinate) is plotted as a function of saccade interval (abscissa). The curve represents equation (2) with $f_{1}(t)$. Same data, curve resulted from model computations of equation (2) with $f_{2}(t)$. Data from one subject.

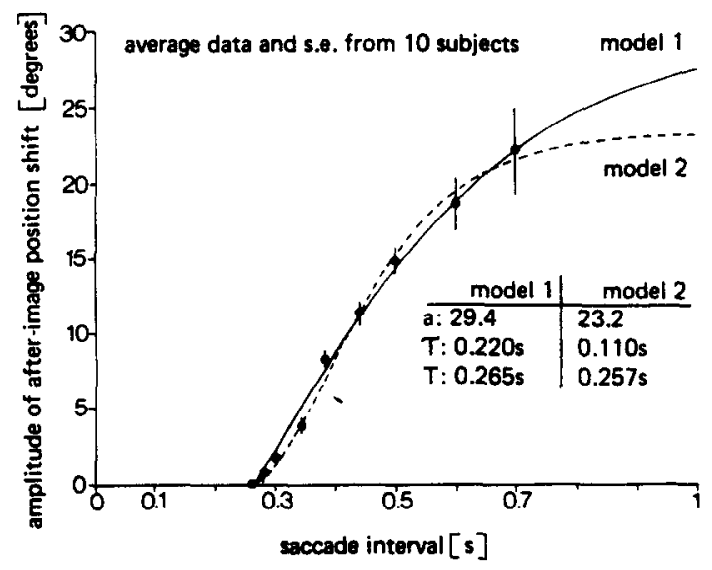

Fig. 8. Same relationship as in Fig. 7. Average data from all 10 subjects. Model computations applying $f_{1}(t)$ (continuous curve) or $f_{2}(t)$ (dashed curve). Constants in equation (2) leading to minimum deviation between experimental data and model predictions are indicated.

The data of four of the ten subjects fitted equation (2) better with $f_{1}(t)$ than with $f_{2}(t)$, while in other subjects the opposite was true. The differences in the sum $Q$ of quadratic deviations between data and the values for the two different models were fairly small in all subjects. For the average data of 10 subjects $f_{2}(t)$ was found to be a better description than $f_{1}(t)$ (Fig. 8). This was not unexpected. Even if $f_{1}(t)$ would have been better than $f_{2}(t)$ in all individual subjects, due to the scatter of $\tau_{1}$ and $T_{1}$ the average data easily led to a curve which is better described by $f_{2}(t)$.

The algebraic means found in the group of 10 subjects were $122 \pm 18 \mathrm{msec}$ (SE) for $\tau_{1}$ and $95 \pm 16 \mathrm{msec}$ for $\tau_{2}$. The corresponding dead times were $T_{1}=290 \pm 9 \mathrm{msec}$ and $T_{2}=256 \pm$ $16 \mathrm{msec}$.

Table 1 exhibits the values obtained for $\tau_{1}, \tau_{2}$, $T_{1}$ and $T_{2}$ in model computations using the experimental data obtained when saccade amplitude was varied [two subjects, Fig. 5(a,b)]. It is evident that $\tau$ or $T$ tended to increase slightly when the saccade amplitude increased to about 25 deg. Above this value however, the saccadic amplitude had no significant effect on $\tau$ and $T$ in these experiments.

\section{DISCUSSION}

After we had completed our investigation, we came across the observation of Köllner (1923) that a foveal afterimage moves less during voluntary quickly repeated saccades performed back and forth horizontally than with slowly 
Table 1. Scaling factor, time constant and dead time of model 1. Data from 2 subjects and 8 different target distances

\begin{tabular}{lcccccccc}
\hline $\begin{array}{c}\text { Target } \\
\text { Distance } \\
\text { (degrees) }\end{array}$ & 13 & 17 & 25 & 38 & 42 & 48 & 55 & 64 \\
\hline $\begin{array}{l}\text { Subject A } \\
a_{1} \text { (deg) }\end{array}$ & 12.3 & 16.5 & 25.5 & 21.9 & 29.2 & 31.0 & 35.1 & 32.7 \\
$\tau_{1}$ (msec) & 53 & 90 & 82 & 82 & 132 & 93 & 122 & 119 \\
$T_{1}$ (msec) & 304 & 320 & 298 & 299 & 289 & 314 & 286 & 287 \\
Subject B & & & & & & & & \\
$a_{1}$ (deg) & 9.0 & 15.8 & 24.7 & 26.9 & 24.9 & 26.5 & 26.6 & 31.6 \\
$\tau_{1}$ (msec) & 49 & 72 & 116 & 115 & 101 & 107 & 82 & 118 \\
$T_{1}$ (msec) & 333 & 333 & 307 & 302 & 302 & 307 & 302 & 303 \\
\hline
\end{tabular}

repeated saccades. With a voluntary saccade alternation frequency of about 3 saccades/sec Köllner saw the foveal afterimages in the dark moving just slightly around a midline position. This observation of Köllner had been preceded by Dittler's (1921) report on a student who observed stability of a foveal afterimage seen in the dark during $7-8 \mathrm{~Hz}$ voluntary horizontal nystagmus. Nagle et al. (1980) and Howard (1984) confirmed this observation, whereby the subjects of Nagle et al. reached an eye movement frequency of $20 \mathrm{~Hz}$ during horizontal oscillopsia. Our present report is a quantitative study of this effect (cf. also Hillebrand, 1920; Hofmann, 1925) and includes the analysis of the states in between low and maximum saccade repetition rates.

At precisely what time relative to the onset of the saccades the process of recalibrating the spatial values of retinal coordinates begins is still a matter for speculation and can not be deduced from our experiments. Purkinje (1825) and Mach (1886) found that the adjustment between retinal coordinates and egocentric localization is slower than the saccades and is completed considerably after the end of a saccade. Our data on the deadtime $T$ and the time constant $\tau$ support this view. Many authors have tried to measure the recalibration time course by determining the perceived spatial location of a small target flashed on the retina before, during or after a saccade (e.g. Matin and Pearce, 1965; Bischof and Kramer, 1969; Matin et al., 1966, 1969, 1970; review in Matin, 1972; Galley, 1974). Some of the data indicate a fairly complex and possible discontinuous process, which depends on saccadic direction towards or away from a self-luminous target in the dark (Mach, 1886; Kaila, 1923; Auersperg and Sprockhoff, 1935). Our present data, however, are very well described by a fairly simple time function analogous to the operation of a one- or two-stage linear filter with a time constant of about $90-120 \mathrm{msec}$, to which a deadtime in the range of $180-330 \mathrm{msec}$ is added. The time constant seems to vary between subjects (Table 1). As compared with the other studies mentioned above, our experimental paradigm has the advantage of being very simple since the visual stimulus is stabilized on the retina and no external visual references are used which might complicate the measurements. Without such an external spatial reference frame the recalibration process of retinal spatial values first reaches its final state after $600-800 \mathrm{msec}$. This state is also characterized by a gain of less than 1 as compared to gain near 1 found when a stationary patterned visual background is present and the afterimage is displaced by saccades across it. As Matin et al. (1982, 1983) demonstrated, afferent retinal signals from parafoveal retinal regions modify the "extraretinal signals", i.e. the "corollary discharge" (Sperry, 1950) or "efference copy" signals (v. Holst and Mittelstaedt, 1950) representing the gaze movements or gaze position. This mechanism, called "visual capture" by Stark and Bridgeman (1983), might also affect the time constant and the deadtime of equations (3) and (4). It is very difficult, however, to measure afterimage position changes during fast back and forth saccades over a well-structured background, since this external visual stimulus becomes spatially unstable at a saccade frequency above 2 saccades/sec and seems to shift back and forth in the opposite direction to the saccadic eye movements (oscillopsia). This is also true of course for a visual reference scale placed in an otherwise dark field of gaze.

Hänsen and Skavenski (1977) applied a new paradigm to measure the accuracy of the eye position signal used for motor control. Their subjects had to perform goal-directed ballistic arm-movements to hit a target which was 
flashed with or without other visual feedback. They found that the hammer blow was fairly accurate in the dark (standard deviations about $1.0 \mathrm{deg}$ ) and concluded that eye position signals are used at gain 1 for this motor control task. Comparing their data and our afterimage pointing results at low alternation rates indicates that the gain provided by efferent or afferent eye position signals is considerably higher for this motor task than for our perceptual task. Hallett and Lightstone $(1976 \mathrm{a}, \mathrm{b})$ measured goaldirected saccades evoked by a small flashed target before, during and after a saccade. For this visual-oculomotor task the response of a second saccade was dependent essentially on spatial coordinates of the target and not on retinal coordinates. This indicates that within the response time for the second saccade, recalibration of the spatial retinal coordinate values was not only correctly performed but also correctly determined backwards in time. It is difficult to compare the data of the rather complex experiments of Hallett and Lightstone with our present data, which are restricted to a fairly "pure" sensory effect as an indicator of efference copy signals, but it is interesting that in the visuo-oculomotor task the recalibration of the spatial retinal coordinate values seems to be performed within the shortest "normal" intersaccadic interval, i.e. within a time of about 150-180 msec.

Obviously our experiments contribute very little to the question of whether mechanoreceptors of the eye muscles, their tendons or the extraocular orbital tissue assist in visual calibration, a subject which has been discussed repeatedly (Sherrington, 1918; Matin et al., 1966; Skavenski, 1972; Skavenski et al, 1972; Brindley et al., 1975; Steinbach and Smith, 1981). It should be emphasized, however, that none of our subjects experienced any movement of the afterimage when the eyeball was moved passively in the orbita at speeds from a few $\mathrm{deg} / \mathrm{sec}$ up to about $300 \mathrm{deg} / \mathrm{sec}$ (cf. also Bell, 1823; Purkinje, 1825).

In addition to the recalibration process of spatial retinal coordinate values, our experiments revealed a second slow perceptual phenomenon as mentioned on p. 221: the doubling of the afterimage observed at a saccade frequency above 2 saccades/sec. There seems to be another space-related process acting in the central visual system which might be important for temporal continuity of pattern and space perception (cf. also MacKay, 1973; Jeannerod et al., 1979). This second process seems to fix the visual signal coordinates in space for about $500 \mathrm{msec}$ after the onset of the saccade, regardless of where the next saccade is directed. This mechanism might be useful in guiding motor activity related to the stable "frame" of the extrapersonal space.

Acknowledgements - The work was supported in part by a grant of the Deutsche Forschungsgemeinschaft (Gr 161). We thank Mrs J. Dames for her assistance in translation of the manuscript and Professor M. Steinbach. York University, Downsview. Toronto, for helpful comments on the manuscript.

\section{REFERENCES}

Aristotle. Parva Naruralia: On Dreams. Text and translation in: Aristotle Works, Vol. VIII, pp. 348-371. Harvard Univ. Press, Cambridge, Heinemann, London. Auersperg A. P. and Sprockhof H. (1935) Experimentelle Beiträge zur Frage der Konstanz der Sehdinge und ihrer Fundierung. Pfügers Arch. ges. Physiol. 236, 301-320.

Baumgartner G. (1977) Neuronal mechanisms of migrainous visual aura. In Physiological Aspects of Clinical Neurology (Edited by Rose C. F.), pp. 111-121. Black. well, Oxford.

Bell C. (1823) On the motions of the eye. Proc. R. Soc. $113,166-186$.

Beyer Th and Grüsser $0 .-1$. (1986) Pointing towards a foveal afterimage at different eye positions. To be published.

Bischof N. and Kramer B. (1968) Untersuchungen und Oberlegungen zar Richtungswahmehmung bei willkürlichen sakkadischen Augenbewegungen. Psychol. Forsch. 32, 195-218.

Bock $O$. and Eckmiller R. (1983) Non-visual control of ingertip position in space: The use of retinal versus oculomotor reference inputs. Soc. Neurosci. Abstr. 9,71.

Brindley G. S., Goodwin G., Kulikowski J. and Leighton D. (1975) Stability of vision with a paralyzed eye. $t$. Physiol, Lond. 253, 65-66.

Brindley G. S. and Lewin W. S. (1968) The sensations produced by electrical stimulation of the visual cortex. J. Physiol. Lond. 196, $479-494$.

Bücking H. and Baumgartner G. (1974) Klinik und Pathophysiologie der initialen neurologischen Symptome bei Migräne (Migraine ophthalmique, Migraine accompagnee). Arch. Psychiat. Nerventheilk. 219, 37-52.

Dittler R. (1921) Ober die Raumfunktion der Netzhaut in ihrer Abhängigkeit vom Lagegefühl der Augen und vom Labyrinth. Z. Sinnesphysiol, 52, 274-310.

Galley N. (1974) Die Richtungswahrnehmung von primäw rem Bild und Hering'schem Nachbild im Veriauf schneller Augenbewegungen. Dr. med. Dissertation, Berlin.

Gottschalk Ch., Grisser O.J. and Lindau M. (1978) Tracking movement of the eyes elicited by auditory stimuli at a constant angular velocity. Pfingers Arch. ges. Physiol. 377, R46.

Grüsser O.-J. (1983) Multimodal structure of the extrapersonal space. In Spatially Oriented Behavior (Edited by Hein A. and Jeannerod M.), pp. 327-357. Springer, Bertin. 
Grüsser O.-J. and Krizič A. (1984) Time constant of perand postsaccadic recalibration of retinal spatial values as measured by a new afterimage method. Invest. Ophthal. visual Sci.. ARVO Suppl 25, 263 P.

Hallett P. E. and Lightstone A. D. (1976) Saccadic eye movements towards stimuli triggered by prior saccades. Vision Res. 16, 99-106.

Hallett P. E. and Lightstone A. D. (1976) Saccadic eye movements to flashed targets. Vision Res. 16, 107-114.

Hansen R. M. and Skavenski A. A. (1977) Accuracy of eye position information for motor control. Vision Res. 17, 919-926.

Hering E. (1861) Vom Ortssinne der Netzhaut. Beiträge zur Physiologie, Heft 1, Leipzig $80 \mathrm{P}$.

Hering E. (1879) Der Raumsinn und die Bewegungen des Auges. In Handbuch der Physiologie (Edited by Hermann L.), Vol. 3, pp. 343-601, Vogel, Leipzig.

Hillebrand F. (1920) Die Ruhe der Objekte bei Blickbewegungen. Jahrb. Psychiat. Neurol. 40, 213-265.

Hofmann F. B. (1925) Die Lehre vom Raumsinn des Auges. Springer, Berlin.

Holst E. von and Mittelstaedt H. (1950) Das Reafferenzprinzip. Wechseiwirkung zwischen Zentralnervensystem und Peripherie. Naturwissenschaften 37, 464-476.

Howard I. P. (1984) Personal communication.

Jeannerod M., Kennedy H. and Magnin M. (1979) Corollary discharge: its possible implication in visual and oculomotor interactions. Neuropsychologia 17, 241-258.

Jolly F. (1902) Über Flimmerskotom und Migraine. Klin. Wschr. Berlin 39, 973-976.

Jung R. (1979) Translokation corticaler Migrainephosphene bei Augenbewegungen und vestibularen Reizen. Neuropsychologia 17, 173-185.

Kaila E. (1923) Die Lokalisation der Objekte bei Blickbewegungen. Psychol. Forsch. 3, 60-77.

Köllner H. (1923) Uber die Abhängigkeit der räumlichen Orientierung von den Augenbewegungen. Klin. Wschr., Berlin 2, 482-484.

Lashley K. S. (1941) Patterns of cerebral integration indicated by the scotomes of migraine. Archs Neurol. Psychiat., Chicago 46, 331-339.

Mach E. (1886) Die Analyse der Empfindungen und das Verhältnis des Physischen zum Psychischen. Fischer, Jena. English translation (1959) The Analysis of Sensations and the Relation of the Physical to the Psychical. Dover. New York.

MacKay D. M. (1973) Visual stability and voluntary eye movements. In Handbook of Sensory Physiology. Vol. VII 3A, Central Processing of Visual Information A. Integrative Functions and Comparative Data (Edited by Jung R.), pp. 307-331. Springer, Berlin.

Matin L. (1972) Eye movement and perceived visual direction. In Handbook of Sensory Physiology (Edited by Jameson D. and Hurvich L.). Springer, Berlin.
Matin L., Matin E. and Pearce D. (1969) Visual perception of direction when voluntary saccades occur. I. Relation of visual direction of a fixation target extinguished before a saccade to a flash presented during the saccade. Percept. Psychophys. 5, 65-80.

Matin L. Matin E. and Pola J. (1970) Visual perception of direction when voluntary saccades occur. II. Relation of visual direction of a fixation target extinguished before a saccade to a subsequent test flash presented before the saccade. Percept. Psychophys. 8, 9-14.

Matin L. and Pearce D. G. (1965) Visual perception of direction for stimuli flashed during voluntary saccadic eye movements. Science, N.Y. 148, 1485-1488.

Matin L., Pearce D. G., Matin E. and Kibler G. (1966) Visual perception of direction: roles of local sign. eye movements and ocular proprioception. Vision Res. 6, 453-569.

Matin L., Picoult E., Stevens J. K. Edwards M. W. Jr. Young D. and MacArthur R. (1982) Oculoparalytic illusion: Visual field dependent spatial mislocations by human partially paralyzed with curare. Science, $N . Y$. 216, 198-201.

Matin L., Steven J. K. and Picoult E. (1983) Perceptual consequences of experimental extraocular muscle paralysis. In Spatially Oriented Behavior (Edited by Hein A. and Jeannerod M.), pp. 243-262. Springer, Berlin.

Nagle M., Bridgeman B. and Stark L. (1980) Voluntary nystagmus, saccadic suppression, and stabilization of the visual world, Vision Res. 20, 717-721.

Purkinje J. (1825a) Beobachtungen und Versuche zur Phisiologie der Sinne II: Neue Beiträge zur Kenntniss des Sehens in subjectiver Hinsicht. Reimer, Berlin.

Purkinje J. (1825b) Uber die Scheinbewegungen, welche im subjektiven Umfange des Gesichtssinnes vorkommen. Bull. der naturwissenschaftichen Sektion der Schlesischen Gesellschaft. IV, pp. 9-10

Richards W. (1971) The fortification illusion of migraine. Scient. Am. 224, 89-96.

Sherrington C. S. (1918) Observations of the sensual role of the proprioceptive nerve supply of the extrinsic eye muscles. Brain 41, 332-343.

Skavenski A. A. (1972) Inflow as a source of extraretinal eye position information. Vision Res. 12. 221-229.

Skavenski A. A., Haddad G. and Steinman R. M. (1972) The extraretinal signal for the visual perception of direction. Percept. Psychophys. 11, 287-290.

Sperry R. W. (1950) Neural basis of the spontaneous optokinetic response produced by visual inversion. $J$. comp. Physiol. Psychol. 43, 482-489.

Stark L. and Bridgeman B. (1983) Role of corollary discharge in space constancy. Percept. Psychophys. 34, 371-380.

Steinbach M. J. and Smith D. R. (1981) Spatial localization after strabismus surgery: Evidence for inflow. Science, N.Y. 213, 1407-1409. 